Sādhanā Vol. 38, Part 6, December 2013, pp. 1065-1066. (C) Indian Academy of Sciences

\title{
Multiphase flows with phase change
}

\section{Foreword}

Multiphase flows with phase change are ubiquitous in many industrial sectors ranging from energy and infra-structure to specialty chemicals and pharmaceuticals. My own interest in multiphase flows with phase change started more than 15 years ago when I had initiated work on riser reactor for fluid catalytic cracking and fluidized bed reactors for poly-propylene. In later years, we encountered multiphase flows with phase change on several occasions while addressing many industrial projects (both at National Chemical Laboratory and at Tridiagonal Solutions, which I co-founded) such as injection of volatile solvents into mass of non-volatile liquid either to remove heat of reaction or to remove impurities; crystallization via anti-solvents (sub or supercritical); boiling in power plants or outside fixed bed reactors; manufacturing of polycrystalline $\mathrm{Si}$; cooling of large forged shafts and so on. In many such industrial projects, there is usually not adequate time and budget to address the problem rigorously. More often than not significant approximations are made based on prior experience and engineering judgement. The need for more rigorous treatment was felt by everyone involved in such projects. This was the background to bring out this special section of 'Sadhana - Academy Proceedings in Engineering Sciences' on 'multiphase flows with phase change'.

The scope of this special section is restricted to multiphase flows with phase change involving gas-liquid (boiling and condensation) and solid-liquid (crystallization/solidification and dissolution/melting) processes. Boiling (and condensation) is relevant and important for variety of areas including electricity generation to chemical reactors. Crystallization is essential to pharmaceutical industries since large numbers of pharmaceutical products are in solid form and are produced by crystallization. Solidification/melting processes are important for many large systems from fast breeder reactors to polymerization reactors. The objective of this special section is to bring together reviews and recent works in these areas to convey differences and similarities in these multiphase flows with phase change. This special section comprises of nine papers which are briefly described here.

Duryodhan et al have reported results of an experimental study of flow boiling through diverging micro- channel. The focus is on understanding the influence of mass and heat flux on boiling in a non-uniform micro-channel. Four flow regimes namely bubbly, slug, slug-annular and periodic dry-out/rewetting were observed. The presented data will be useful to provide insights into boiling in diverging micro-channels.

Sardeshpande and Ranade have briefly reviewed two phase flow boiling in small channels. Published information, on flow regimes (with and without boiling), heat transfer, pressure drop, critical heat flux and film thickness for channels smaller than $3 \mathrm{~mm}$ is critically reviewed. An attempt is made to identify strengths and weaknesses of published approaches and computational models for simulating boiling in small channels. 
Biswas has presented variants of volume of fluid methods for surface tension dominant two phase flows. A combined level set and volume of fluid (CLSVOF) method was developed and used to simulate bubble growth in water at near critical pressure for different degrees of superheat. Simulations were used to understand frequency of bubble formation, deviation from periodic release and structure of resulting flows.

Sikarwar et al have developed mathematical model of drop-wise condensation on textured surfaces. The model incorporates different scales: formation of drops at atomistic scale, droplet growth, coalescence, slide-off and fall-off. It was found that drop instability controls the heat transfer coefficient in drop-wise condensation. The simulations were used to investigate the influence of fluid properties, surface inclination and wettability on droplet size distribution, heat transfer coefficient and wall shear stress.

Bagul et al reviewed entrainment phenomena in gas-liquid two phase flow. Though this paper does not deal directly with the phase change, the entrainment from the gas-liquid surface is an important concern in many of the applications involving liquid to gas phase change (boiling). In this paper, various experimental, analytical and numerical investigations addressing entrainment/carry over are critically analysed to bring out a coherent theme.

Petiti et al in their paper entitled 'CFD modelling of condensers for freeze drying process' discuss the development of a computational tool for simulating condensation processes. CFD models were used to understand flow and condensation as well as deposition of ice in the condenser. They have investigated laboratory scale and an industrial scale condenser. The models and tools developed in this work will be useful to enhance performance of freeze dryers.

Sudha and Velusamy have presented numerical analysis of grid plate melting after a core disruptive accident in a fast breeder reactor. The focus is on estimating time scale of melt through grid plate so that thermal load on the core catcher plate can be estimated.

Gudibande and Iyer have developed a modified enthalpy method for simulation of melting and solidification. They have reported development of a new cell splitting method which avoids some arbitrary constants (called mushy zone constants) typically used in the enthalpy method. Besides eliminating these arbitrary constants, the method was shown to eliminate waviness present in the classical enthalpy method. This method is demonstrated for both one and two-dimensional melting problems with and without natural convection.

Vedantam and Ranade provide a review of key thermodynamic, kinetic and hydrodynamic aspects of crystallization. Published information and modelling studies on crystallization bring out these three aspects are critically reviewed. Efforts are made to discuss emerging modelling tools needed for better designs of crystallization processes.

I would like to express my sincere appreciation to the contributors of this special section on 'multiphase flows with phase change'. Each of the authors has provided perspective and highlighted challenges from their respective application areas. I hope that this issue will stimulate further work on this very important area from industrial as well as from scientific point of view.

December 2013

VIVEK V RANADE (Guest Editor)

Industrial Flow Modelling Group, Chemical Engineering and Process Development Division, CSIR - National Chemical Laboratory, Pune 411008, India email: vv.ranade@ncl.res.in 